\title{
AVALIAÇÃO DE POPULAÇÕES DE MARACUJAZEIRO-DOCE (Passiflora alata Curtis) OBTIDAS DE POLINIZAÇÃO ABERTA ${ }^{1}$
}

\author{
MOISÉS RODRIGUES MARTINS², JOÃO CARLOS DE OLIVEIRA³, ANTONIO ORLANDO DI MAURO³, PAULO \\ CÉSAR DA SILVA
}

RESUMO - Catorze características agronômicas de cinco populações de maracujazeiro-doce (Passiflora alata Curtis) foram avaliadas em JaboticabalSP, no período de abril de 2000 a março de 2001. Número de frutos por planta (NF), número de sementes/fruto (NS), massa do fruto (MF) e produção (PRO) foram caracteres que apresentaram os mais elevados índices de variabilidade entre plantas, possibilitando, assim, a seleção de plantas superiores quanto a estes caracteres. Médias dos caracteres produção (PRO), número de frutos/planta (NF), espessura da casca (EC), número de sementes/fruto (NS) e rendimento em polpa (\%P), analisadas pelo teste de Tukey, a 5\% de probabilidade, não apresentaram diferença significativa entre as populações. Observou-se considerável variabilidade entre plantas e baixa variabilidade entre as populações nestes caracteres.

Termos para indexação: variabilidade fenotípica, maracujá, Passiflora alata

\section{EVALUATION OF SWEET PASSION FRUIT (PASSIFLORA ALATA CURTIS) POPULATIONS OBTAINED BY OPEN POLINIZATION}

ABSTRACT - Fourteen horticultural characteristics of five sweet passion fruit (Passiflora alata Curtis) populations were evaluated in JaboticabalSP, from April 2000 to March 2001. A considerably high variability among plants and low among populations was observed for these characters. The number of fruits per plant (NF), number of seeds/fruit (NS), fruit mass (MF) and production (PRO) were the characters with the highest levels of variability among plants, thus, allowing then for the selection of superior plants for these characters. Averages of production (PRO), number of fruits/ plant (NF), rind thickness (EC), number of seeds/fruit (NS) and pulp yield (\%P), compared by the Tukey test at 5\% probability, did not present significant differences among the populations.

Index terms: phenotipic variability, passion fruit, Passiflora alata

\section{INTRODUÇÃo}

Passiflora alata Curtis, conhecida popularmente como maracujá-doce, é cultivada no Brasil pela sua elevada cotação no mercado de frutas frescas, pois a polpa é muito saborosa e doce. A planta é utilizada também como ornamental, pelas suas flores grandes e vistosas, além de sua utilização como planta medicinal (Vasconcelos \& Cereda 1994; Oliveira et al. 1994).

Oliveira et al. (1982), estudando as características de Passiflora alata de cinco diferentes procedências, observaram que o peso médio dos frutos variou de 89,32 g a 195,92 g. Já o comprimento e a largura variaram de $6,85 \mathrm{~cm}$ a $10,13 \mathrm{~cm}$ e 4,75 a $6,93 \mathrm{~cm}$, respectivamente. $\mathrm{O}$ número de sementes/fruto variou de 143 a 276; sendo a espessura do mesocarpo de $7 \mathrm{~mm}$ a $10 \mathrm{~mm}$ e $15,28^{\circ}$ teor de sólidos solúveis totais (Brix). Meletti et al. (1992) caracterizaram algumas introduções de germoplasma de maracujazeiros em Jundiaí-SP, encontrando frutos de $P$. alata com peso médio de 128,3 g, 19,5 g de polpa, largura e comprimento do fruto de $6,6 \mathrm{~cm}$ e $8,9 \mathrm{~cm}$, respectivamente, espessura da casca de $15,50 \mathrm{~mm}$, rendimento em polpa de $15 \%$, teor de sólidos solúveis totais de $15,1^{\circ}$ Brix e 224 sementes/fruto. Já Vasconcelos (1991) obteve, em Botucatu-SP, valores de peso dos frutos de $156 \mathrm{~g}$ a $320 \mathrm{~g}$; comprimento do fruto de $9,32 \mathrm{~cm}$ a $12,73 \mathrm{~cm}$; largura do fruto de $6,97 \mathrm{~cm}$ a $8,79 \mathrm{~cm}$; $75,84 \%$ de casca e $24,16 \%$ de polpa; 18,80 a $21,20^{\circ}$ Brix e 159 a 317 sementes/fruto. Algumas lavouras de maracujá-doce, segundo Piza Júnior (1998), produzem frutos que chegam a atingir $16 \mathrm{~cm}$ de comprimento e a pesar quase 500 gramas. $O$ mesmo autor cita que os produtores de maracujá-doce já estão multiplicando vegetativamente plantas produtivas, com frutos grandes, com mais de 350 gramas de peso e de cor amareloalaranjada.

Segundo Oliveira \& Ferreira (1991), o que se busca em maracujazeiro é um genótipo produtivo, vigoroso, resistente às pragas e doen- ças, de ampla adaptação, frutos grandes com alto teor de sólidos solúveis no suco e de cor amarela, resistente ao transporte e armazenamento. De acordo com Meletti et al. (1992), o gênero Passiflora apresenta ampla variabilidade genética para ser explorada, observando-se grandes variações no florescimento, produtividade, em caracteres do fruto, resistência a pragas e doenças.

Os maracujazeiros são espécies alógamas, de cultivo recente, com ampla variabilidade genética a ser explorada (Oliveira et al., $1982 \mathrm{e}$ Bruckner, 1997). No entanto, pesquisas relacionadas com a área de genética são escassas.

Em função do exposto, objetiva-se com este estudo caracterizar e analisar geneticamente cinco populações de maracujazeiro-doce de forma a obter informações que possam ser utilizadas em programas de melhoramento.

\section{MATERIALEMÉTODOS}

O experimento abrangeu cinco populações originadas de sementes de polinização aberta. Três destas foram pré-selecionadas da coleção do Banco Ativo de Germoplasma (BAG) da Faculdade de Ciências Agrárias e Veterinárias/UNESP, em Jaboticabal-SP, denominadas de JAB1, JAB2 e JAB3, e duas provenientes de pomares comerciais da região de Ribeirão Preto-SP e Valinhos-SP, denominadas, respectivamente, de RPe VAL. O experimento foi instalado na Área Experimental do Departamento de Produção Vegetal da FCAV/UNESP, Câmpus de Jaboticabal-SP e ocupou uma área $2.520 \mathrm{~m}^{2}$, adotando-se um espaçamento de $6 \mathrm{~m}$ entre plantas na fileira e 3,5 m entre fileiras, em um delineamento em blocos casualizados com 4 repetições. A unidade experimental constituiu-se de 6 plantas, num total de 24 plantas por população. A semeadura foi realizada em casa de vegetação, em sacos plásticos, no dia 10-02-99 e transplantadas em 18-03-99. Depois de um perío-

\footnotetext{
(Trabalho 127/2002). Recebido: 24/04/2002. Aceito para publicação: 06/03/2003. Parte da Tese de Doutorado do primeiro autor, apresentada à FCAV-UNESP Câmpus de Jaboticabal - SP

2 Aluno de doutorado do Curso de Pós-Graduação em Genética e Melhoramento de Plantas, da FCAV-UNESP - Via de acesso Paulo Donato Castellane S/N, 14884900 - Jaboticabal - SP, e-mail: moises64@zipmail.com.br

${ }^{3}$ Profs. Titulares do Depto. de Produção Vegetal da FCAV-UNESP - Via de acesso Paulo Donato Castellane S/N, 14884-900 - Jaboticabal - SP. fitotec@ fcav.unesp.br ${ }^{4}$ Aluno de doutorado do Curso de Pós-Graduação em Genética e Melhoramento de Plantas, da FCAV-UNESP - Via de acesso Paulo Donato Castellane S/N, 14884900 - Jaboticabal - SP, e-mail: pcsilva@ netsite.com.br
} 
do de aclimatação, o plantio definitivo ocorreu em 21-06-99. A condução das plantas foi realizada em espaldeira com um fio de arame $n^{\circ} 10$ a 1,80 m do solo, com poda de formação para induzir brotações laterais. Dois ramos secundários, em cada lado do ramo principal, seguiram-se então pelo arame de sustentação, dando origem aos ramos terciários ou produtivos. Os tratos culturais seguiram as recomendações para o maracujá-amarelo, adotando-se as práticas rotineiramente realizadas no Câmpus da UNESP/Jaboticabal.

Os caracteres agronômicos avaliados neste estudo foram: comprimento do fruto $(\mathrm{CF})$, largura do fruto $(\mathrm{LF})$, número de sementes/fruto (NS), espessura da casca (EC), diâmetro longitudinal da cavidade ovariana (DLC), diâmetro transversal da cavidade ovariana (DTC), sólidos solúveis totais (SST), massa do fruto (MF), massa da polpa (MP), massa da casca $(\mathrm{MC})$, rendimento em polpa $(\% \mathrm{P})$, número de frutos/planta, produção/planta (PRO) e tempo decorrido da abertura da flor à colheita dos frutos (TAC).

Para a avaliação destes caracteres, frutos foram coletados quinzenalmente e levados ao laboratório, onde foram pesados e posteriormente contado o número de sementes em cada fruto. As características de comprimento e massa foram medidas por meio de paquímetro e balança de precisão de $0,1 \mathrm{~g}$, respectivamente. Os teores de sólidos solúveis totais (SST) foram determinados pela leitura direta em um refratômetro manual com precisão de 0,20 , expresso em graus Brix. A quantificação da produção das populações foi obtida de abril/2000 a março/2001, a intervalos regulares, na freqüência de duas vezes por semana. Foram coletados dados de produção por planta, em número e peso de frutos. Para análise do tempo decorrido da abertura da flor à colheita (TAC), flores foram marcadas no dia da abertura e acompanhadas semanalmente, observando-se o tempo decorrido até a colheita.

As análises de variância foram efetuadas, usando-se o programa estatístico SAS e as médias das populações foram comparadas pelo teste de Tukey, a 5\% de probabilidade. As estimativas dos coeficientes de correlação foram calculadas pelo método de Pearson descrito em Steel \& Torrie (1960), usando-se o programa SAS.

\section{RESULTADOSE DISCUSSÃO}

Na Tabela 1, são apresentadas as médias, os valores máximos, valores mínimos e desvios-padrão dos catorze caracteres agronômicos avaliados. Observou-se que o comprimento do fruto (CF) apresentou média geral, entre todas as populações estudadas, de 108,99 mm, com o máximo de $152,90 \mathrm{~mm}$ apresentado pela população RP e mínimo de 84,30 apresentada pela população JAB3. A largura do fruto (LF) variou de $56,10 \mathrm{~mm}$ a $93,40 \mathrm{~mm}$, ambos no acesso JAB3, com média geral de $74,76 \mathrm{~mm}$. Estes valores são menores que os encontrados por Vasconcelos (1991), em Botucatu-SP, porém maiores que os encontrados por Melleti (1992), em Jundiaí-SP e Oliveira (1982), em Jaboticabal. Na Tabela 2, observa-se que estes caracteres encontram-se altamente correlacionados com a espessura da casca (EC), diâmetro longitudinal da cavidade ovariana (DLC), diâmetro transversal da cavidade ovariana (DTC), massa do fruto (MF) e massa da casca (MC), porém não se correlacionam com o rendimento em polpa $(\% \mathrm{P})$, indicando que frutos maiores não necessariamente terão maior rendimento de polpa.

TABELA 1- Valores máximos, mínimos, média e desvio-padrão de catorze caracteres agronômicos de cinco populações de maracujazeiro-doce (Passiflora alata), obtidos de polinização aberta, avaliadas em Jaboticabal-SP, no período de abril de 2000 a março de 2001.

\begin{tabular}{|c|c|c|c|c|c|c|c|c|c|c|c|c|c|c|c|}
\hline \multirow{2}{*}{ Pop. } & \multirow{2}{*}{ Valor } & \multicolumn{14}{|c|}{ CARACTERES AVALIADOS } \\
\hline & & CF & LF & NS & EC & DLC & DTC & SsT & MF & MP & MC & $\% \mathrm{P}$ & NF & PRO & TAC \\
\hline \multirow{3}{*}{ JAB1 } & Máx & 129,60 & 92,00 & 344,20 & 14,90 & 67,30 & 98,10 & 21,00 & 331,32 & 95,24 & 268,06 & 39,10 & 331,00 & 62,91 & 63,00 \\
\hline & Min & 84,70 & 59,40 & 116,50 & 7,60 & 41,50 & 64,70 & 15,90 & 136,88 & 27,33 & 99,21 & 19,09 & 38,00 & 6,91 & 57,00 \\
\hline & DP & 14,18 & 9,05 & 70,16 & 1,89 & 6,20 & 8,54 & 1,18 & 54,67 & 17,50 & 44,56 & 5,65 & 96,71 & 15,89 & 1,72 \\
\hline \multirow{2}{*}{ JAB2 } & Máx & 118,10 & 82,80 & 359,60 & 15,00 & 57,30 & 89,10 & 20,70 & 298,81 & 79,80 & 258,23 & 32,22 & 296,00 & 65,13 & 64,00 \\
\hline & DP & 6,26 & 4,21 & 54,01 & 1,33 & 3,26 & 5,48 & 1,17 & 35,10 & 11,58 & 32,47 & 4,98 & 74,17 & 14,34 & 1,54 \\
\hline \multirow{4}{*}{ JAB3 } & Máx & 118,60 & 93,40 & 379,60 & 13,90 & 62,90 & 88,40 & 20,20 & 362,62 & 73,30 & 302,54 & 45,74 & 338,00 & 56,94 & 64,00 \\
\hline & Min & 84,30 & 56,10 & 197,90 & 7,50 & 42,90 & 65,20 & 17,10 & 153,02 & 34,46 & 86,93 & 16,56 & 7,00 & 1,43 & 59,00 \\
\hline & Média & 102,74 & 70,62 & 283,76 & 10,8 & 49,96 & 74,96 & 18,44 & 198,04 & 55,96 & 142,08 & 29,00 & 124,04 & 20,87 & 61,00 \\
\hline & DP & 12,17 & 10,13 & 44,47 & 1,78 & 4,93 & 7,25 & 0,80 & 48,02 & 11,11 & 45,39 & 6,62 & 95,35 & 15,75 & 1,80 \\
\hline RP & Máx & 152,90 & 90,30 & 437,70 & 16,10 & 65,00 & 107,70 & 20,20 & 367,61 & 91,57 & 276,04 & 34,97 & 313,00 & 69,87 & 66,00 \\
\hline \multirow{4}{*}{ VAL } & Máx & 148,00 & 89,90 & 393,00 & 15,20 & 65,00 & 109,60 & 19,60 & 368,19 & 97,78 & 294,19 & 42,61 & 346,00 & 53,76 & 57,00 \\
\hline & Min & 93,10 & 65,50 & 165,40 & 8,70 & 42,70 & 65,20 & 16,80 & 155,40 & 36,64 & 101,77 & 18,84 & 10,00 & 18,78 & 51,00 \\
\hline & Média & 116,49 & 76,54 & 262,14 & 11,44 & 55,38 & 83,65 & 17,92 & 226,86 & 63,88 & 162,98 & 28,68 & 118,81 & 23,60 & 54,00 \\
\hline & DP & 14,69 & 6,24 & 57,44 & 1,48 & 5,69 & 10,61 & 0,78 & 43,08 & 16,78 & 46,96 & 6,81 & 84,93 & 16,07 & 1,92 \\
\hline \multicolumn{2}{|c|}{ Média geral } & 108,99 & 74,76 & 263,25 & 11,22 & 53,16 & 79,24 & 18,08 & 214,65 & 57,69 & 156,96 & 27,27 & 138,00 & 25,02 & 60,01 \\
\hline
\end{tabular}

CF- comprimento do fruto(mm); LF- largura do fruto (mm);NS-número de sementes/fruto; EC-espessura da casca (mm); DLC-diâmetro longitudinal da cavidade ovariana (mm); DTC-diâmetro transversal da cavidade ovariana(mm); SST-sólidos solúveis totais( $\left.{ }^{\circ} \mathrm{Brix}\right)$; MF-massa do fruto (g); MP-massa da polpa(g); MC-massa da casca $(\mathrm{g})$; \% P- rendimento em polpa; NF-número de fruto/planta; PRO-produção(kg/planta); TAC-tempo decorrido da abertura da flor a colheita do fruto (dias).

O número médio de sementes foi de 263 , com uma variação de 116,50 a 437,70 sementes, encontradas nas populações JAB1 e RP, respectivamente. Essa variação foi maior do que a encontrada por Vasconcelos (1991), em Botucatu-SP, e Oliveira et al. (1982), em Jaboticabal-SP. O número de sementes correlacionou-se, neste estudo, positivamente com diâmetro longitudinal da cavidade ova- riana (DLC), diâmetro transversal da cavidade ovariana (DTC), massa da polpa (MP) e porcentagem da polpa (\%P), porém não apresentou correlação com a espessura da casca (Tabela2). Isto sugere que frutos de maracujazeiro-doce devem ser selecionados para ampliação da cavidade ovariana, para obter-se maior rendimento em polpa. 
Quanto à produção, houve uma grande amplitude de variação, encontrando-se plantas com produção muito baixa, como no acesso JAB3 (1,43 kg/planta), e plantas com alta produção, como na população RP $(69,87 \mathrm{~kg})$. Esta característica apresentouse correlacionada apenas com o número de frutos (NF) e massa do fruto (MF) não tendo correlação com o tempo decorrido da abertura da flor a colheita (TAC).

O teor de sólidos solúveis totais (SST), variando de 15,70 a $21,00{ }^{\circ}$ Brix, apresentados pelos acessos RP e JAB 1, e o tempo decorrido da abertura da flor à colheita (TAC), variando de 51 a 66 dias, apresentado pelos acessos VAL e RP (Tabela 1), apresentaram-se pouco correlacionadas com as demais características avaliadas no estudo, mostrando serem caracteres pouco dependentes. O rendimento em polpa $(\% \mathrm{P})$ das populações avaliadas está em torno de 27,27\%, variando de 13,57 a 45,74\%. Estes valores aproximam-se dos encontrados por Vasconcelos (1991) em Botucatu-SP. Notou-se pequenas variações relativas a sólidos solúveis totais (SST) e espessura da casca (EC), o que torna difícil o critério de seleção, utilizando-se estes caracteres.

TABELA 2- Estimativas dos coeficientes de correlação de Pearson entre os pares de caracteres estudados, em cinco populações de Passiflora alata, no período de abril de 2000 a março de 2001. Jaboticabal-SP.

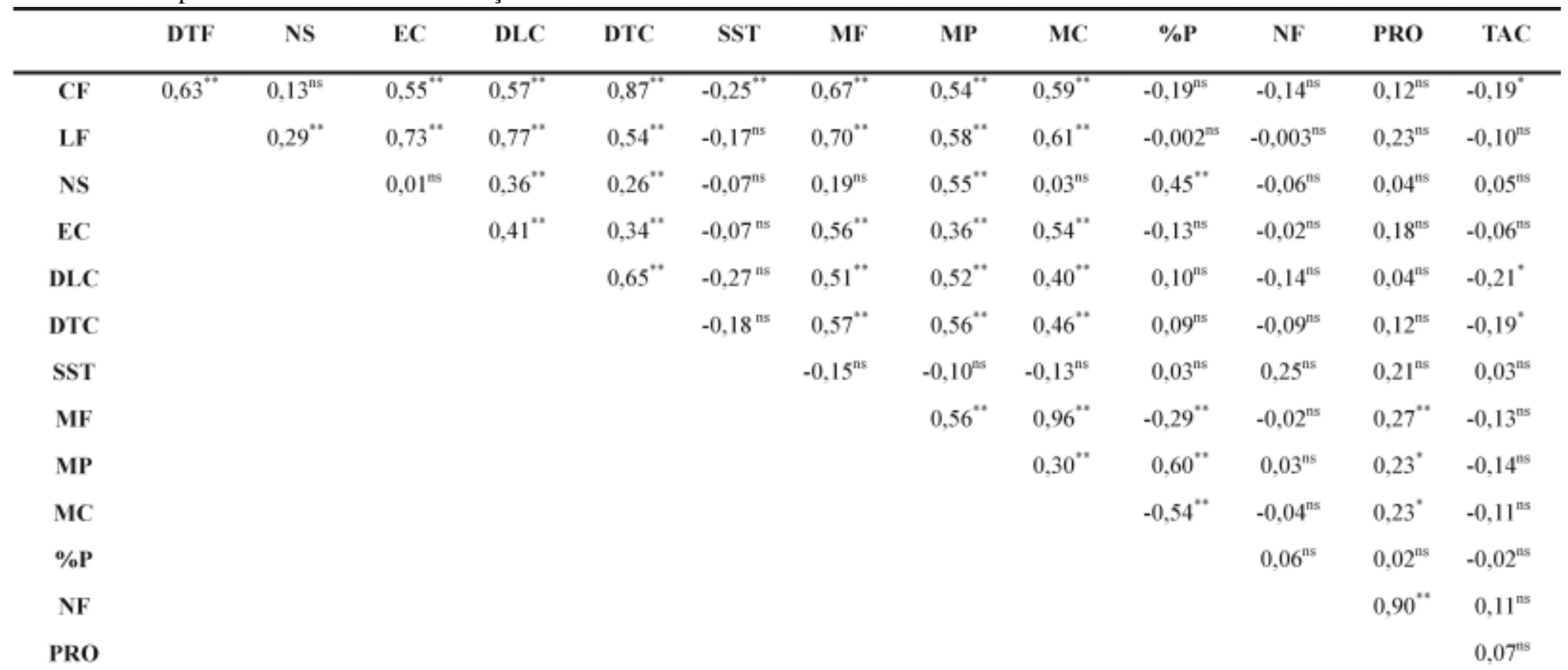

CF- comprimento do fruto(mm); LF- largura do fruto (mm); NS-número de sementes/fruto; EC-espessura da casca (mm); DLC-diâmetro longitudinal da cavidade ovariana (mm); DTC-diâmetro transversal da cavidade ovariana(mm); SST-sólidos solúveis totais( ${ }^{\circ}$ Brix); MF-massa do fruto (g); MP-massa da polpa(g); MC-massa da casca $(\mathrm{g})$; \% P-rendimento em polpa; NF-número de fruto por planta; $\mathrm{PRO}$-produção(kg/planta); TAC - tempo decorrido da abertura da flor à colheita do fruto (dias); ns-não significativo; $* *, *$ significativo a $1 \%$ e $5 \%$ de probabilidade, respectivamente.

Na Tabela 3, mostra-se a comparação das médias e o coeficiente de variação geral das populações estudadas. Não se observou diferença significativa entre as médias das cinco populações para espessura da casca (EC), rendimento em polpa (\%), número de frutos/planta (NF) e produção (PRO); entretanto, número de fru- tos/planta (NF) e produção (PRO) apresentaram alta variabilidade entre plantas. Os coeficientes de variação $(\mathrm{CV})$ para número de frutos (NF) e produção (PRO) foram muito altos, demonstrando grande variabilidade entre plantas, constituindo-se em parâmetros a serem utilizados em processos seletivos.

TABELA 3- Comparação de médias, pelo teste de Tukey, das cinco populações e coeficiente de variação dos caracteres avaliadas. Jaboticabal-SP.

\begin{tabular}{ccccccccccccccc}
\hline & CF & LF & NS & EC & DLC & DTC & SST & MF & MP & MC & $\%$ P & NF & PRO & TAC \\
\hline JAB1 & $105,8 \mathrm{~b}$ & $75,0 \mathrm{ab}$ & $234,7 \mathrm{~b}$ & $11,1 \mathrm{a}$ & $53,6 \mathrm{abc}$ & $78,4 \mathrm{ab}$ & $18,0 \mathrm{ab}$ & $208,2 \mathrm{ab}$ & $54,8 \mathrm{ab}$ & $153,4 \mathrm{ab}$ & $26,4 \mathrm{a}$ & $158,4 \mathrm{a}$ & $26,7 \mathrm{a}$ & $61,0 \mathrm{a}$ \\
JAB2 & $104,2 \mathrm{~b}$ & $73,1 \mathrm{ab}$ & $263,1 \mathrm{ab}$ & $10,9 \mathrm{a}$ & $51,3 \mathrm{bc}$ & $75,8 \mathrm{~b}$ & $18,3 \mathrm{ab}$ & $202,7 \mathrm{~b}$ & $52,1 \mathrm{~b}$ & $150,6 \mathrm{ab}$ & $25,9 \mathrm{a}$ & $158,1 \mathrm{a}$ & $27,2 \mathrm{a}$ & $62,0 \mathrm{a}$ \\
JAB3 & $102,7 \mathrm{~b}$ & $70,6 \mathrm{~b}$ & $283,7 \mathrm{a}$ & $10,8 \mathrm{a}$ & $49,9 \mathrm{c}$ & $74,9 \mathrm{~b}$ & $18,4 \mathrm{a}$ & $198,0 \mathrm{~b}$ & $55,9 \mathrm{ab}$ & $142,0 \mathrm{~b}$ & $29,0 \mathrm{a}$ & $124,0 \mathrm{a}$ & $20,8 \mathrm{a}$ & $61,0 \mathrm{a}$ \\
RP & $116,0 \mathrm{a}$ & $78,6 \mathrm{a}$ & $272,5 \mathrm{ab}$ & $11,8 \mathrm{a}$ & $55,6 \mathrm{a}$ & $83,5 \mathrm{a}$ & $17,6 \mathrm{~b}$ & $238,4 \mathrm{a}$ & $62,0 \mathrm{ab}$ & $176,3 \mathrm{a}$ & $26,3 \mathrm{a}$ & $129,9 \mathrm{a}$ & $26,7 \mathrm{a}$ & $61,0 \mathrm{a}$ \\
VAL & $116,4 \mathrm{a}$ & $76,0 \mathrm{a}$ & $262,1 \mathrm{ab}$ & $11,4 \mathrm{a}$ & $55,3 \mathrm{ab}$ & $83,6 \mathrm{a}$ & $17,9 \mathrm{ab}$ & $226,8 \mathrm{ab}$ & $63,8 \mathrm{a}$ & $162,9 \mathrm{ab}$ & $28,6 \mathrm{a}$ & $118,8 \mathrm{a}$ & $23,6 \mathrm{a}$ & $54,0 \mathrm{~b}$ \\
\hline CV\% & 8,6 & 8,8 & 20,5 & 12,6 & 9,2 & 9,4 & 5,1 & 18,7 & 22,2 & 22,7 & 18,9 & 55,7 & 56,8 & 3,2 \\
\hline
\end{tabular}

CF- comprimento do fruto(mm); LF- largura do fruto (mm); NS-número de sementes/fruto; EC-espessura da casca (mm); DLC-diâmetro longitudinal da cavidade ovariana (mm); DTC-diâmetro transversal da cavidade ovariana(mm); SST-sólidos solúveis totais $\left({ }^{\circ}\right.$ Brix); MF-massa do fruto (g); MP-massa da polpa(g); MC-massa da casca (g); \%P-rendimento em polpa; NF-número de fruto por planta; PRO-produção (kg/planta); TAC-tempo decorrido da abertura da flor a colheita do fruto (dias). Médias, de uma mesma coluna, seguidas da mesma letra não diferem significativamente, ao nível de 5\% de probabilidade, pelo teste de Tukey. $\mathrm{CV}(\%)$ - coeficiente de variação. 
$\mathrm{Na}$ Tabela 4, encontram-se as estimativas dos componentes de variância dos caracteres estudados, e têm-se os componentes da variância e sua participação porcentual na variância total. Observa-se que os caracteres número de sementes/planta (NS), massa do fruto (MF), número de frutos/planta (NF) e produção (PRO), características importantes para seleção, apresentaram alta variabilidade entre plantas, o que pode pos- sibilitar ganhos relativos, desde que se processe a seleção a partir deles. O componente de variância da população $\left(\sigma^{2} p\right)$ teve baixa participação na variância no total, o que permite inferir que não é aconselhável a seleção de maracujazeiro-doce, com base só nesse critério, e alguns caracteres apresentaram valores elevados para o componente de variância entre bloco x população $\left(\sigma^{2} b^{*} p\right)$.

TABELA 4- Componentes de variância nos caracteres estudados e participação percentual dos componentes na variância total das cinco populações de Passiflora alata. Jaboticabal - SP.

\begin{tabular}{|c|c|c|c|c|c|c|c|c|c|c|c|c|c|c|}
\hline & $\mathrm{CF}$ & LF & NS & $\overline{E C}$ & DLC & DTC & SST & MF & MP & MC & $\% \mathrm{P}$ & NF & PRO & TAC \\
\hline$\sigma^{2} \mathbf{b}$ & 0 & 8,08 & 72,16 & 0,07 & 0 & 0 & 0 & 318,08 & 33,37 & 167,89 & 3,00 & 1077,92 & 7038,12 & 0 \\
\hline$\sigma^{2} \mathbf{p}$ & 13,65 & 4,81 & 31,70 & 0 & 4,09 & 8,33 & 0,005 & 56,41 & 13,20 & 0 & 0 & 0 & 0 & 9,39 \\
\hline$\sigma^{2} \mathbf{b}^{*} \mathbf{p}$ & 103,70 & 8,65 & 545,13 & 0,66 & 3,71 & 24,51 & 0,25 & 607,08 & 20,16 & 525,98 & 6,73 & 1206,35 & 6971,53 & 0 \\
\hline$\sigma^{2} \mathrm{pl}$ & 92,28 & 43,78 & 2993,69 & 2,05 & 23,98 & 55,35 & 0,88 & 1615,21 & 165,95 & 1265,72 & 26,53 & 5881,57 & 19706,74 & 3,79 \\
\hline$\sigma^{2} t$ & 209,62 & 65,32 & 3642,68 & 2,78 & 31,78 & 88,19 & 1,13 & 2596,66 & 232,69 & 1959,59 & 36,26 & 8165,84 & 36595,65 & 13,18 \\
\hline$p p \sigma^{2} b$ & 0 & 12,36 & 1,98 & 2,52 & 0 & 0 & 0 & 12,24 & 14,34 & 8,57 & 8,27 & 13,20 & 19,23 & 0 \\
\hline $\mathbf{p p} \sigma^{2} \mathbf{p}$ & 6,50 & 7,36 & 0,87 & 0 & 12,87 & 9,45 & 0,44 & 2,18 & 5,67 & 0 & 0 & 0 & 7,86 & 71,24 \\
\hline$p p \sigma^{2} b^{\star *} p$ & 49,47 & 13,24 & 14,96 & 23,64 & 11,67 & 27,79 & 22,12 & 23,37 & 8,66 & 26,84 & 18,56 & 14,77 & 19,05 & 0 \\
\hline $\mathrm{pp} \sigma^{2} \mathrm{pl}$ & 44,03 & 67,04 & 82,19 & 73,74 & 75,46 & 62,76 & 77,88 & 62,21 & 71,33 & 64,59 & 73,17 & 72,05 & 53,86 & 28,76 \\
\hline
\end{tabular}

CF- comprimento do fruto(mm); LF- largura do fruto (mm); NS-número de sementes/fruto; EC-espessura da casca (mm); DLC-diâmetro longitudinal da cavidade ovariana (mm); DTC-diâmetro transversal da cavidade ovariana(mm); SST-sólidos solúveis totais( $\left.{ }^{\circ} \mathrm{Brix}\right)$; $\mathrm{MF}-\mathrm{massa}$ do fruto (g); MP-massa da polpa(g); MC-massa da casca (g); \%P-rendimento em polpa; NF-número de fruto/planta; PRO-produção(kg/planta); TAC-tempo decorrido da abertura da flor a colheita do fruto (dias); $\delta \mathrm{b}$ variância de blocos; $\sigma^{2}$ p variância de populações; $\sigma^{2} b^{*}$ p variância da interação bloco populações; $\sigma^{2}$ pl variância de plantas; $\sigma^{2} t$ variância total; pp $\sigma^{2} b$ variância da participação porcentual de blocos; $p p \sigma^{2}$ p variância da participação porcentual de populações; $p p \sigma^{2} b^{*} p$ variância da participação porcentual da interação blocos populações; $p$ p $\sigma^{2}$ pl variância da participação porcentual de plantas.

\section{CONCLUSÕES}

Os resultados obtidos na presente pesquisa permitiram as seguintes conclusões:

1) A grande variabilidade na maioria dos caracteres avaliados permite a seleção preliminar de plantas com características agronômicas promissoras.

2) Os caracteres número de frutos/planta (NF) e produção (PRO) apresentaram elevados índices de variabilidade entre plantas, podendo ser amplamente utilizados em um processo seletivo.

3) Não foi possível selecionar genótipos através do componente de variância-população.

4) A seleção baseada nos caracteres para o componente de variância entre bloco x população mostrou dificuldade em função de alguns valores elevados apresentados.

\section{REFERÊNCIAS BIBLIOGRÁFICAS}

BRUCKNER, C.H. Perspectivas do melhoramento genético do maracujazeiro In: SÃO JOSÉ, A.R. et al. Maracujá: temas selecionados (1) Porto Alegre: Cinco continentes, 1997. p. 25-46.

MELETTI, L.M.M.; SOARES-SCOT, M.D.; PINTO-MAGLIO, C.A.F.; MARTINS, F.P. Caracterização de germoplasma de maracujazeiro (Passiflora sp). Revista Brasileira de Fruticultura, Cruz das Almas, v.14, n. 2, p.157-62, 1992.

OLIVEIRA, J. C.; SALOMÃO, T.A.; RUGGIERO, C. Variações observa- das em frutos de Passiflora alata Ait. In: CONGRESSO BRASILEIRODEOLERICULTURA, 21., CONGRESSODASOCIEDADEBRASILEIRA DE FLORICULTURA E PLANTAS ORNAMENTAIS, 2., 1981, Campinas. Proceedings... p.343-345.

OLIVEIRA, J.C., FERREIRA, F.R. Melhoramento genético do maracujazeiro. In: SÃO JOSÉ, A.R. A cultura do maracujá no Brasil. Vitória da Conquista-BA: UESB, 1991. p.211-239.

OLIVEIRAJ.C.; NAKAMURA,K.; MAURO,A.O.; CENTURION, M.A.P. da C. Aspectos gerais do melhoramento do maracujazeiro. In: SÃO JOSÉ, A.R. (Ed.). Maracujá: produção e mercado. Vitória da Conquista-BA: UESB-DFZ, 1994. p. 27-28.

PIZA JÚNIOR, C.T. A cultura do maracujá na região Sudeste do Brasil. In: SIMPÓSIO BRASILEIRO SOBRE ACULTURADO MARACUJAZEIRO, 5., 1998, Jaboticabal. Anais... Jaboticabal: FCAV/UNESP, 1998. p. 20-48.

STEEL, R.G.D.; TORRIE, J.H. Principles and procedures of statistics. New York: Mc Graw-Hill, 1960. 418p.

VASCONCELOS, M. A. Biologia floral do maracujá-doce (Passiflora alata Dryand) nas condições de Botucatu. 1991. 98f. Dissertação (Mestrado em Agronomia - Área de Horticultura) - Faculdade de Ciências Agronômicas, Universidade Estadual Paulista, Botucatu, 1991.

VASCONCELOS, M.A.; CEREDA, E. O cultivo de maracujá-doce. In: São José, A.R. maracujá: produção e mercado. Vitória da ConquistaBA: UESB-DFZ, 1994. p.71-81. 